\title{
La polémica sobre el entrenamiento psicológico del Comprehensive soldier fitmess
}

\author{
García Silgo, M. ${ }^{1}$
}

Sanid. mil. 2014; 70 (1): 40-48; ISSN: 1887-8571

\begin{abstract}
RESUMEN
Antecedentes: En enero de 2011, la revista de Psicología American Psychologist, publicación oficial de la Asociación Americana de Psicología, publicó un monográfico sobre el programa de entrenamiento Comprehensive Soldier Fitness desarrollado por el ejército de los Estados Unidos. Este programa contempla el entrenamiento de diversas variables, entre otras, psicosociales. Dicha publicación suscitó cinco réplicas de psicólogos civiles del ámbito académico norteamericano, comenzando así una polémica determinada por las acusaciones que se hicieron a dicho programa de entrenamiento, al equipo de psicólogos involucrados, a la Asociación Americana de Psicología y a la psicología militar. Objetivos: Presentar un resumen de las réplicas, la contrarréplica de Martin E.P. Seligman, un análisis crítico de la polémica y dar continuidad al debate planteado. Método: El análisis de las réplicas se realiza desde la ética profesional y la reflexión, teniendo en cuenta la psicología basada en la evidencia. Conclusiones: Mientras que algunas réplicas promueven un debate moralista y parten de determinadas ideologías políticas, otras suscitan un debate interesante. Sus autores dudan de la ética en la psicología militar y sugieren la no asociación de la psicología positiva en estos contextos. Sin embargo, hacer esto supondría una falta de ética y, por tanto, una paradoja. La polémica plantea una reflexión necesaria para la psicología.
\end{abstract}

PALABRAS CLAVE: Comprehensive Soldier Fitness, Psicología Militar, Ética profesional, Dilemas sociales, Conflicto de rol.

\section{The controversial about the Comprehensive Soldier Fitness training program}

SUMMARY: Background: In January 2012, the journal American Psychologist from the American Psychological Association published a report about the United States Army Comprehensive Soldier Fitness training program. This program has a section on psychosocial variables. The special elicited five replies from academic civilian American psychologists, which prompted controversial allegations of the Army psychological fitness program, the psychology team involved the American Psychologist Association and the military psychology. Objectives: The aim of this article is to raise an overview of the comments, to show the Seligman's response to the comments, to make a summary of the controversy and to give continuity to the debate. Methods: This analysis has been done by using the code of ethics and the reflection from the evidence based practice in psychology. Conclusions: While some of the criticisms show a partial view based on some political ideologies, promoting a moral debate instead of a scientific psychology one, others open an interesting debate. The authors of the reviews doubt the military psychology ethics and suggest the no association between positive psychology and the Army. However, doing this would be unethical and therefore a paradox. The controversy raises a necessary question for psychology.

KEYWORDS: Comprehensive Soldier Fitness, Military psychology, Professional ethics, Social dilemmas, Role conflict.

\section{ANTECEDENTES}

En enero de 2011, la revista de psicología American Psychologist, publicación oficial de la American Psychological Association (APA; Asociación Americana de Psicología), con un factor de impacto para el año 2011 de 3.121 , publicó un monográfico sobre un programa de entrenamiento desarrollado por el ejército de los Estados Unidos. Dicho programa, originalmente denominado Comprehensive Soldier Fitness (CSF; entrenamiento integral del soldado) y posteriormente conocido como Comprehensive Soldier and Family Fitness (CSF2; entrenamiento integral del soldado y las familias) ${ }^{2}$, se ha difundido en medios es-

${ }^{1}$ Cap. Psicólogo. Unidad de Apoyo Psicológico. Inspección General de Sanidad de la Defensa. Madrid. España.

Dirección para correspondencia: Mónica García Silgo. Unidad de Apoyo Psicológico. Inspección General de Sanidad de la Defensa. Madrid. España. Telf.: 914222968. mgarsil@et.mde.es

Recibido: 19 de diciembre de 2012

Aceptado: 17 de septiembre de 2013 pañoles en distintas ocasiones ${ }^{3-7}$ por lo que no se detallará aquí. Esta publicación de la APA suscitó cinco réplicas de psicólogos civiles del ámbito académico norteamericano (Eidelson, Pilisuk y Soldz ${ }^{8}$; Phipps ${ }^{9}$; Krueger ${ }^{10}$; Dyckman ${ }^{11}$; Campbell Quick ${ }^{12}$ ) y, lejos de estancarse en la propia publicación que los presentó, se vieron propagadas por distintos medios de comunicación y páginas web. Se puso así en entredicho la estrategia en la que el gobierno de los Estados Unidos había invertido, al menos, 125 millones de dólares ${ }^{13}$ para la preparación psicológica de los militares norteamericanos.

El alcance del asunto lo determinan las graves críticas que se hicieron al CSF, al equipo de psicólogos involucrados, a la APA y a la psicología militar en general. Polémicas como ésta trascienden cuando la opinión pública se apropia de los argumentos infundados y convierten una confrontación de opiniones entre colegas, en una crítica social.

Desde el punto de vista de la psicología militar operativa hay que reconocer que el CSF es el programa más significativo de promoción de salud mental y entrenamiento bio-psicosocial basado en la evidencia jamás desarrollado e implantado 


\section{La polémica sobre el entrenamiento psicológico del Comprehensive soldier fitness}

en la historia de los ejércitos. Aunque no ha sido el primer programa, ni el único ${ }^{3}$, se encuentra entre los pioneros en destacar la importancia que tiene el estigma asociado a los problemas mentales en la prevención y promoción de la salud mental de los militares.

\section{Antecedentes al Comprehensive Soldier Fitness}

Desde que en el año 1998, la psicología positiva (en dónde se enmarcan los estudios de resiliencia) fuera reconocida públicamente por Martin E.P. Seligman ${ }^{14}$, las investigaciones sobre resiliencia psicológica han ido cobrando relevancia, aunque el interés científico por este tema sea muy anterior a $1998^{4}$. Publicaciones divulgativas de distintos tipos han contribuido a popularizar el término «resiliencia». Sin embargo, hay que denunciar que, en ocasiones, el concepto se está divulgando en la sociedad por personal no experto en el área o no especializado en la profesión o a través de textos de «autoayuda», lo que puede estar dando lugar a una banalización del término y a una importante confusión de sus principios, objetivos y métodos. De hecho, gran parte de las críticas que se le pueden estar haciendo a la psicología positiva, están relacionadas con esta cuestión.

Desde hace décadas, la tendencia por parte de destacados autores así como distintos organismos internacionales y nacionales, ha sido trasladar la atención de la enfermedad y las características negativas de las personas desde un punto de vista biológico, a la salud y lo positivo desde un punto de vista bio-psico-social (p. ej. ver citas ${ }^{15-22}$ ). En algunos países, como los Estados Unidos, incluso se han creado leyes para promover la resiliencia de la Nación ${ }^{23}$. Además, desde el comienzo de los conflictos asimétricos, la resiliencia del personal militar es una prioridad para las estrategias de Defensa de distintos países (p. ej. National Security Research Division ${ }^{24}$ ).

Ya años antes al inicio del CSF, la salud mental de los militares norteamericanos desplegados fue reconocida públicamente, desarrollándose importantes investigaciones ${ }^{25}$ y equipos de trabajo multidisciplinares, como el Mental Health Advidsory Team (MHAT). De este modo, en la última década se ha avanzado considerablemente en la información disponible sobre salud mental y variables psicosociales en operaciones militares.

El Mando militar también ha manifestado interés por el tema. El trigésimo sexto General Jefe de Ejército (JEME) de los Estados Unidos, George W. Casey Jr., declaró que los esfuerzos por mejorar la salud mental del personal desplegado no estaban siendo suficientes y ordenó que se implementaran programas de entrenamiento de tipo integral (incluyendo variables psicosociales y dándoles la misma importancia que a las variables físicas). Otra acción destacable realizada por este JEME en materia de salud mental, fue reconocer oficialmente -y por primera vez en el entorno castrense- la existencia del estigma asociado a los problemas mentales y la necesidad de hacerle frente ${ }^{13}$.

Así, el intento de poner solución al problema psicosocial (suicidios, divorcios, ansiedad, depresión, Trastorno de Estrés Postraumático-TEPT-, abuso de sustancias, problemas de pareja, problemas con la ley, etc.) derivado de los despliegues en Irak y Afganistán, condujo a los líderes militares de los Estados Unidos a buscar ayuda en la psicología, encargan- do a Seligman y su equipo de la Universidad de Pensilvania (UPENN) esta labor ${ }^{26}$. La colaboración del mundo científicoacadémico y militar se materializó el 1 de octubre de 2008, en el programa $\mathrm{CSF}^{27}$.

\section{Origen del Comprehensive Soldier Fitness}

El CSF surgió para abordar las alarmantes estadísticas de problemas psicosociales asociados a los despliegues en Irak y Afganistán, con el objetivo de promocionar la prevención primaria (desde el punto de vista de la salud pública ${ }^{28}$ ) o secundaria (desde el punto de vista de la psicología de las organizaciones $^{29}$ ). Ambos tipos de prevención pretenden lo mismo, dirigir las intervenciones a fortalecer a la población y así protegerla ante posibles problemas mentales e interpersonales. Para ello, los programas deben fomentar el desarrollo de los factores de protección de la población. De este modo, el CSF es un programa continuado de entrenamiento de las variables moduladoras que tienen la capacidad de disminuir y/o anular los efectos de los estresores psicosociales asociados a la profesión militar. Dichas variables son los factores que componen la resiliencia de los militares en el plano psicológico, físico, social, espiritual y familiar.

Conviene aclarar que el módulo psicológico del CSF está basado en la evidencia de estudios validados desde el ámbito científico académico (UPENN) y científico militar (Walter Read Army Institute of Research) previos al CSF (para una ampliación ver cita $^{3}$.

\section{DESCRIPCIÓN DE LA POLÉMICA EN TORNO AL COMPREHENSIVE SOLDIER FITNESS}

Como se comentó en el apartado de antecedentes, en octubre de 2011, la revista American Psychologist publicó cinco replicas escritas por siete autores (Eidelson, Pilisuk y Soldz ${ }^{8}$; Phipps; krueger ${ }^{10}$; Dyckman ${ }^{11}$ y Campbell Quick ${ }^{12}$ ) en las que se criticaba el programa CSF -presentado en enero de 2011 por la misma revista-, a la APA, a los psicólogos que participaron en el CSF, a la psicología militar y a la política exterior de los Estados Unidos. En el mismo número de octubre de 2011, Seligman ${ }^{30}$ respondió $^{2}$ a las réplicas. A continuación se muestra un resumen, agrupado por temáticas, de lo que se dijo por parte de todos los autores involucrados en la polémica.

\section{Interpretación del CSF como una violación al Código de Núremberg}

Eidelson, Pilisuk y Soldz ${ }^{8}$ exponen que «la única preocupación que surgió, en todo el especial de la revista American Psychologist, fue que no era ético negar el CSF a los soldados». Estos autores consideran que el CSF «carece de ética por ser un programa masivo en el que no hay consentimiento informado» $\mathrm{y}$ recuerdan que «es una seria violación al Código de Núremberg establecido a finales de la II Guerra Mundial en respuesta a los experimentos nazis». 


\section{Críticas a la política exterior norteamericana, a la guerra, al ejército y a la psicología militar}

Eidelson, Pilisuk y Soldz ${ }^{8}$ creen que «ser patriótico no es apropiado para psicólogos que se dedican a promover la salud y el bienestar universales»; que «los Estados Unidos no emplean su ejército en defensa propia, sino en ejercer un control imperial para sus intereses nacionales» y que «no se puede apoyar a una institución a estar del lado de la guerra sin cuestionarlo cuidadosamente».

En el especial de enero de 2011 de la revista de la APA, Seligman y Fowler ${ }^{26}$ afirmaron que «la psicología no debería ayudar a la política exterior de los Estados Unidos». Ante esto, Phipps ${ }^{9}$ responde diciendo que: «sería maravilloso que la psicología ayudara a dar forma a la política exterior de los Estados Unidos» y critica que los autores del monográfico acepten ciegamente la premisa del ejército de que «esta guerra interminable es inevitable». Además, entiende que el CSF «es un experimento colosal diseñado para dar apoyo a los militares americanos en la lucha contra la ideología yihadista» y se plantea si «jes esto psicología positiva?» y «ipor qué no una psicología positiva que cuestione a los líderes que nos dicen que el uso de la fuerza es inevitable y que busque soluciones pacíficas en lugar de ayudarles?».

Krueger ${ }^{10}$ propone que «traer la psicología al ejército es usar las herramientas de la psicología para una industria»; que «el cliente del CSF es el ejército, no los soldados individualmente» y que «lo que le interesa al ejército es estandarizar la conducta de sus miembros y, para ello, puede castigar». De lo que concluye que, «aunque desde una perspectiva grupal pueda ser necesario y legítimo», no cree que «sea legítimo que los psicólogos obstaculicen el conflicto de intereses entre el ejército y los soldados y luego actúen como si se preocuparan por el bienestar de éstos».

Dyckman ${ }^{11}$ cuenta que se quedó «decepcionado al ver todo un especial de la American Psychologist dedicado a la psicología militar»y «especialmente preocupado por las justificaciones de una sola moral presentadas por Seligman y Fowler ${ }^{26}$ en el artículo final de la revista». Para él, los autores del monográfico «infravaloraron las posibles críticas a su programa y descartaron los daños inherentes de la cooperación entre la psicología y el ejército» y cree que «hay que hacer una distinción moral muy compleja». Además, ante las pretensiones de Seligman y Fowler ${ }^{26}$ de que «ayudar al ejército a hacer que los que se ganan la vida matando se sientan mejor y puedan dignificar su trabajo» él responde que «haciendo un repaso de los últimos 50 años, su ejército ha estado casi siempre ayudando a mantener brutales dictaduras en otros países» y se pregunta «¡en qué casos está justificado que se pueda ayudar a alguien a sentirse mejor por matar?». Al final de su exposición, señala que «está extremadamente preocupado por la unión entre la psicología y lo militar» porque, dice, «hay psicólogos ayudando a desarrollar técnicas de interrogatorio (p.ej. torturas)».

En el monográfico de la APA, Seligman y Fowler ${ }^{26}$ comentaron que «tres ideologías (fascismo, comunismo e islamismo yihadista) nacidas en el pasado siglo, intentan derrocar la democracia por la fuerza» y añadieron que «sin un ejército fuerte, sin el empleo responsablemente de la fuerza y en defensa propia, sus victorias no habrían tenido lugar y la defensa contra las amenazas presentes y futuras sería imposible». Ante esto, Dickman ${ }^{11}$ expone que hay dos hechos que no son evidentes: «el primero es que la fuerza se haya usado siempre responsablemente y, el segundo, es que se esté actuando en defensa propia, ya que Irak no atacó a los Estados Unidos el 11-S». El autor también comenta que «Seligman y Fowler ${ }^{26}$ ignoraron el hecho obvio de que la mejor manera de prevenir el TEPT es, en primer lugar, evitando que se produzca el trauma». Además, critica que «los psicólogos implicados en el CSF no consideren que se esté luchando en guerras innecesarias».

\section{Críticas a la moral de la APA y a la metodología y principios del CSF}

Eidelson, Pilisuk y Soldz ${ }^{8}$ expresan que «en la creación del CSF, no se ha partido de revisiones ni programas piloto». Además, extraen uno de los objetivos del CSF «aumentar el número de soldados que den significado y tengan un crecimiento tras su experiencia en combate» planteados por Cornum, Matthews y Seligman ${ }^{27}$ para criticar que «el programa no aprecia el valor de las emociones negativas como la ira, el miedo, y la culpa» y que «el CSF desprecia las duras realidades sociales como la pobreza o la opresión». Basándose en la crítica a la psicología positiva de «por ejemplo Coyne y Tennen ${ }^{31}$ » señalan que «se promocionan unos principios sin suficiente soporte científico» y concluyen que «la falta de crítica al CSF, por parte de la APA, revela mucho acerca de la moral a la que se enfrenta la profesión».

Para Phipps ${ }^{9}$ y Campbell Quick ${ }^{12}$ «todo el monográfico fue sobre prevención secundaria, sin una sola palabra sobre prevención primaria, cuando la intervención preferente, desde el punto de vista de la salud pública, es el de la prevención primaria». Phipps ${ }^{9}$, por su parte, se plantea «si la psicología puede hacer mejores soldados y si se puede usar la resiliencia y la inoculación al estrés para inmunizar a los soldados contra el estrés de la guerra». El autor defiende que «la perspectiva de crear soldados psicológicamente invulnerables le parece aterradora».

Dyckman ${ }^{11}$ cree que «el CSF se presentó como un programa para prevenir el sufrimiento pero no presentaron evidencias del programa» y opina que «no está claro que cualquier entrenamiento pueda preparar adecuadamente a los soldados para la realidad del sufrimiento que han presenciado y que ellos mismos han infligido en otros seres humanos».

Krueger ${ }^{10}$ señala que «los autores del monográfico de American Psychologist justifican su compromiso con el ejército argumentando que la psicología científica ha sido relevante sobre todo en las guerras mundiales» y añade que «el desarrollo de herramientas válidas lleva tiempo, hacer una lista de resultados deseables no es suficiente, la psicología aplicada debe tener objetivos y es criticable que todos los soldados tengan que pasar por el CSF».

En cuanto al módulo del CSF que interviene en la espiritualidad, Phipps ${ }^{9}$ apunta que «le parece obsceno manipular la espiritualidad humana para propósitos militares».

\section{Respuesta de Seligman a las críticas}

Eidelson, Pilisuk y Soldz ${ }^{8}$ afirman que «el CSF es una investigación masiva» y que «atenta contra el Código de Núrenberg, el 


\section{La polémica sobre el entrenamiento psicológico del Comprehensive soldier fitness}

consentimiento informado y el código ético». Pero, según Selig$\operatorname{man}^{30}$ «el CSF no se encuadra dentro de la definición de investigación según la regulación del Ejército 70-25 (Uso de voluntarios como sujetos de investigación, 1990, p. 17)». Este autor explica que «el CSF tiene estatus de programa de entrenamiento» y que «al igual que entrenan físicamente por las mañanas, todos los soldados asisten a clases donde se les enseña cómo reconocer los signos del posible suicidio y el abuso sexual, cómo resistir psicológicamente cuando se es capturado por el enemigo o porqué usar el cinturón de seguridad al conducir». El autor concluye que «estos programas no requieren consentimiento informado».

También le acusan de no hacer investigación piloto. Sin embargo, tal y como apunta el autor ${ }^{30}$ «el ejército lo eligió basándose en un extensivo estudio piloto en civiles y, en concreto, 17 estudios controlados y replicados de prevención de ansiedad y depresión $^{32}$ (mayores componentes del TEPT)». Además, añade que «era el único programa de resiliencia en el que el entrenamiento de los entrenadores se había validado en una muestra militar ${ }^{33}$ ». Para Seligman ${ }^{30}$ «hacer estudios piloto habría estado bien», sin embargo, comenta que «el ejército lo rechazó porque habría llevado años hacerlo, estaban en mitad de una guerra y el programa era el más replicado de la literatura científica».

Seligman $^{30}$ señala, también, que sus críticos dijeron que «el entrenamiento en resiliencia podría, por ejemplo, dañar a los soldados». Sin embargo, defiende que «en el Congreso de los Estados Unidos se declaró que 'tras analizar los datos del CSF se observa que no hay evidencias de que el CSF cause daño' (Office of Comprehensive Soldier Fitness, 2011)». Además, apunta que «para determinar la eficacia del programa CSF se está haciendo un robusto análisis de éste. Evaluación que está siendo mucho más detallada y rigurosa científicamente que otros programas de entrenamiento del ejército»y añade que «resultados preliminares del CSF indican que los soldados que participan en el CSF, en comparación con un grupo control: Están en mejor forma emocional; se adaptan mejor; tienen menos pensamientos catastrofistas cuando se enfrentan a la adversidad; emplean sus fortalezas del carácter o sus puntos fuertes más a menudo; muestran mejores habilidades de afrontamiento; son más optimistas; y tienden a percibir más el apoyo que el ejército presta a las necesidades de sus familias.»

Seligman $^{30}$ dice que Krueger ${ }^{10}$ le acusa de que las pruebas se hicieron rápidamente «pero el GAT... ( Global Assessment Tool; es el instrumento de evaluación del CSF) replica Seligman ${ }^{30} \ll \ldots$ no se desarrolló descuidadamente. Se consultaron expertos, se eligieron ítems de medidas ya probadas, se comprobó el nivel de lectura y se pasaron los ítems a muestras piloto muy grandes de soldados. Tras el análisis de ítems y el análisis de factores, se redujo el test a un número manejable de ítems. El resultado fueron sub-escalas con buena consistencia interna. E1 89\% de los ítems ya habían sido publicados en la literatura revisada». Además, explica Seligman ${ }^{30}$, «hay dos informes técnicos que muestran que hay una relación significativa entre las puntuaciones del GAT y conducta negativa (suicidio, violencia criminal, uso de drogas ilícitas) y conducta positiva (carrera profesional)» y aclara que «pronto se presentaran más resultados».

Seligman ${ }^{30}$ comenta, también, que «no acepta ciegamente la realidad y no está en la posición de evaluar si es probable que se siga en estado de guerra en el futuro cercano, pero existe una posibilidad de que así sea.» Como posibilidad, cree que «el ejército debe estar preparado para ello y el CSF es un camino basado en la evidencia para preparar a los soldados a hacer frente a esa posibilidad»; que «todos los demás comentarios de desaprobación de la política exterior americana quieren renegar del conocimiento que la psicología tiene en este campo» y se pregunta si «irenegarían también de sus habilidades como profesionales cuando los soldados vayan a sus consultas en busca de tratamiento?».

Los coautores del monográfico de la revista de la APA de enero de 2011 y Seligman «están orgullosos de poder ofrecer su conocimiento y sus habilidades a los soldados americanos, esperan cariñosamente que estas intervenciones funcionen y se beneficien, están a favor de la paz y la solución de los conflictos $»^{30}$ y señalan que «esto es lo que está presente en el Congreso y la Casa Blanca» ${ }^{30}$. Además, entienden que «su ejército lleva a cabo las órdenes de sus políticos, por lo que los militares se merecen lo mejor que la psicología puede ofrecer en prevención y tratamiento» ${ }^{30}$.

Para acabar, Seligman ${ }^{30}$ termina su réplica con dos asuntos. El primero es que «no le parece inocuo invocar el Código de Núremberg y la violación ética en este contexto». «Estas alegaciones» para él, «infundadas, tienen un efecto espeluznante para muchos miembros de su profesión, que quieren ayudar a sus soldados, así como para su gobierno» y se pregunta si «¿está siendo acusado de violaciones éticas?». Cuenta que una de las razones por las que sus colaboradores y él propusieron un número entero en la American Psychologist dedicado a este tema, fue para «alentar a los psicólogos a acudir en ayuda del gobierno» y cree que «es urgente que los psicólogos no se desanimen». El segundo asunto es que ante las acusaciones de «abrazar ciegamente nociones simples de patriotismo», alega que «su patriotismo, lejos de estar ciego, ve a los Estados Unidos como el país que dio a sus abuelos, perseguidos de muerte en Europa, un sitio seguro en el que ver crecer a sus hijos y nietos, y al Ejército de América como la fuerza que se interpuso entre las cámaras nazis de gas y él», por lo que «cuenta sus días con los Sargentos y Generales como los más completos de su vida».

\section{ANÁLISIS DE LA POLÉMICA}

Los autores de la polémica emplearon argumentos de diversa índole, abriendo un debate entre profesionales y suscitando ciertas dudas acerca de la credibilidad del programa de salud mental creado por el ejército de los Estados Unidos, el equipo de la UPENN y que, según Casey ${ }^{13}$ y Gottman, Gottman y Atkins ${ }^{34}$, cuenta con el apoyo del Congreso de los Estados Unidos.

Parece que en las réplicas al monográfico de la revista de la APA de enero de 2011, se unen creencias políticas y morales para abatir un programa de promoción y prevención de salud mental $\mathrm{y}$, de paso, una profesión, la psicología militar. Esta posible ausencia de imparcialidad puede haber partido del propio monográfico que dio origen a las réplicas, en cuyo fondo se observan matices propios de la cultura estadounidense (p.ej., exteriorización pública del patriotismo, apoyo incondicional a su ejército, etc.). En este punto hay que recordar que la imparcialidad es una obligación, para todos, en la profesión (ver artículo 15 del Códi- 
go Deontológico del Psicólogo establecido en el Estado Español; ver también criterio 1.02 «Conflictos entre la Ética y la Ley, las normativas y otras Autoridades Legales Gubernamentales» de los Principios Éticos y el Código de Conducta de los Psicólogos de la APA).

En las réplicas, abundan dos tipos de argumentos. En los primeros, se extraen algunos de los principios postulados por el CSF, se desvirtúan y después se justifica el rechazo al programa y al modelo teórico que hay tras él. Por ejemplo, decir que «el programa no aprecia el valor de las emociones negativas como la ira, el miedo y la culpa» ${ }^{8}$, que «el CSF desprecia las duras realidades sociales como la pobreza o la opresión $»^{8}$, o que «se promocionan unos principios sin suficiente soporte científico» ${ }^{8}$, parecen comentarios desvirtuados para dirigir la crítica, inevitablemente, a una única conclusión posible. En los segundos, basándose en una moral determinada o en un hecho no citado (como es que se diga que «hay psicólogos ayudando a desarrollar técnicas de interrogatorio» en las torturas) ${ }^{11}$, se concluye que la psicología en el ejército es una manipulación del bienestar y la salud a favor de las guerras. Ambos argumentos parecen estar sesgados, ya que tienden al catastrofismo, extraen conclusiones en ausencia de o con evidencias contrarias, y perciben la situación -el programa CSF y la psicología militar- en función de actitudes personales.

Para facilitar el análisis y la reflexión, las réplicas se han agrupado en dos bloques diferenciados. Uno trata sobre la psicología positiva y la psicología positiva en el ejército y otro sobre la ética en la psicología militar.

\section{La psicología positiva en el ejército}

El planteamiento de Phipps ${ }^{9}$ sobre si se está usando la psicología positiva para luchar contra la ideología yihadista carece de rigor científico y, por otra parte, cabe preguntarse si ¿Debería la psicología positiva asumir el cometido de cuestionar a los líderes políticos de que el uso de la fuerza es inevitable o de que lo correcto sea buscar soluciones pacíficas como pretende este autor? ¿No es esto desproporcionado y pretencioso para la profesión?

Cuando Eidelson, Pilisuk y Soldz ${ }^{8}$ dicen que «el CSF promociona unos principios sin suficiente soporte científico», se basan en la crítica de Coyne y Tennen ${ }^{31}$ a la psicología positiva para justificar su opinión. Sin embargo, Coyne y Tennen ${ }^{31}$ atacan este enfoque mediante una crítica restringida a algunos principios de la psicología positiva en pacientes de cáncer. Además, Eidelson, Pilisuk y Soldz ${ }^{8}$ omitieron que Aspinwall y Tedeschi ${ }^{35}$ ya habían advertido de los riesgos que hay en asociar conceptos erróneos, pero populares, de la psicología positiva con el cáncer $(21 / 01 / 2010)$ con anterioridad a la publicación de Coyne y Tennen $^{31}(10 / 02 / 2010)$.

En la misma línea de Eidelson, Pilisuk y Soldz ${ }^{8}$ sobre que «el CSF no valora las emociones negativas» otra autora ${ }^{36}$ ha señalado que la promoción del optimismo y las emociones positivas en el CSF, podría suponer una fuente de presión perjudicial para los soldados que realmente presenten emociones negativas tras un largo periodo de despliegue, creando un efecto iatrogénico y provocando una no recuperación o una recuperación desadaptada. Sin embargo, algunos de los resultados del modelo positivo aplicado al personal militar ya se han publicado ${ }^{2,37-39}$ y no se aprecian efectos negativos asociados al programa CSF. Además, el estudio del optimismo como recurso psicológico cuenta con una trayectoria y ha resultado ser un buen predictor de la salud y enfermedad ${ }^{40}$. Por otra parte, ciertos problemas psicosociales y trastornos mentales, como por ejemplo el TEPT, son variables organizacionales asociadas al ejército y así quedó constatado por decisión judicial ${ }^{41,42}$, por lo que buscar maneras de afrontar estos problemas debe ser un objetivo prioritario en las políticas de salud mental que se planeen para esta población.

Otra fuente de presión -señalada implícitamente por Eidelson, Pilisuk y Soldz ${ }^{8}$ - podría ser el planteamiento que se hace del crecimiento postraumático ${ }^{43}$ como un objetivo del $\mathrm{CSF}^{44}$. Aunque Tedeschi y McNally ${ }^{44}$ explican los fundamentos teóricos tras dicho planteamiento, no queda claro cómo se puede trasladar un objetivo privado o personal de estas características a uno público o impuesto desde fuera.

Dyckman ${ }^{11}$ afirma que «no está claro que cualquier entrenamiento pueda preparar adecuadamente a los soldados para la realidad del sufrimiento...» manifestando así, cierto desconocimiento hacia el programa CSF, ya que éste no es «cualquier entrenamiento», sino uno consolidado y basado en la evidencia.

Phipps $^{9}$ y Campbell Quick ${ }^{12}$ critican que «el CSF es un programa de prevención secundaria», pero no argumentan cómo han llegado a dicha conclusión. De hecho, entre los mismos artículos de los que han extraído esa idea, se encuentran referencias que indican que la prevención del CSF es más primaria que secundaria ${ }^{26,27,45,46}$. Otros autores ${ }^{47-50}$ han explicado como es el modelo de prevención que respalda el CSF.

En cuanto a «la manipulación obscena de la espiritualidad para propósitos militares» que plantea Phipps ${ }^{9}$, hay que recordar que las intervenciones en la espiritualidad desde un punto de vista religioso, existen en los ejércitos antes que la psicología o el CSF (lo cual no es una defensa, ya que esto no se hacía bajo el amparo de una ciencia, como si pretende hacer el CSF). Salvo excepciones (p. ej. críticas a monjes budistas en Japón por enseñar estrategias de meditación a militares tras la II Guerra Mundial ${ }^{51,52}$ ) no se ha propuesto que las intervenciones espirituales en los ejércitos sean perversas. Por otra parte, el que se haya demostrado que la espiritualidad -no tanto desde un punto de vista religioso, aunque a veces pueda serlo- sea una variable moduladora de la salud y el bienestar de las personas $^{53-57}$, dato confirmado también en personal militar46, ${ }^{58-61}$, es una referencia objetiva que puede tenerse en cuenta en los programas de resiliencia. Quizás sea el hecho de que el programa CSF se imparta indiscriminadamente en la población militar, lo que podría resultar ser una intromisión en las creencias espirituales y convertirse, por tanto, en una estrategia poco ética. Dado que son los aspectos religiosos o espirituales los que han sido y son objeto de manipulación evidente en algunas regiones del mundo (p. ej., extremismo islámico) se debe ser especialmente prudente para no adoctrinar en estos aspectos íntimos. Como apuntan Pargament y Mahoney ${ }^{62}$ los psicólogos están obligados a atender esta dimensión y a reducir los posibles sesgos cuando tratan a clientes con distintos pasados religiosos. En cualquier caso, la dimensión espiritual del CSF que se presenta en el monográfico de enero de 2011 de la APA $^{63}$ no parece estar constreñida a ninguna ideología o religión, ni aparenta esconder matices manipulativos. Basta leer el artículo 


\section{La polémica sobre el entrenamiento psicológico del Comprehensive soldier fitness}

de Pargament y Sweeney ${ }^{63}$ para entender la concepción de la espiritualidad en el CSF.

Para acabar, ante las objeciones sobre la psicología positiva en los programas de salud mental en los ejércitos, cabe decir que, antes del Battlemind Training (programa de entrenamiento de la resiliencia en el que se basa el CSF; para una ampliación ver $\operatorname{cita}^{3}$ ) nunca se había creado un programa de entrenamiento -basado en la evidencia- que preparara psicológicamente para el combate ${ }^{64}$. Antes de éste, había experiencias preventivas, pero éstas no habían tenido un impacto significativo en la resiliencia de los militares, como por ejemplo los programas de mantenimiento de la adaptación y el reconocimiento del desajuste emocional ${ }^{48} \mathrm{o}$ las sesiones psicoeducativas de una hora en fases predespliegue ${ }^{48,65}$.

\section{La ética de la psicología en el ejército}

Krueger, autor de numerosas publicaciones sobre cómo los intereses colectivos entran en conflicto con los privados (dilemas sociales), cree que «existe un conflicto de intereses entre el ejército y los soldados» tipo dilema del prisionero (El dilema del prisionero es un ejemplo clásico que se emplea para explicar la Teoría de los juegos. Para una ampliación ver p.ej. $\operatorname{citas}^{66-68}$ ). Pero esta es una interpretación teórica de una realidad que bien podría no representar la población total que compone los ejércitos. El mismo autor va más allá de esta opinión y añade que «no es cierto que los psicólogos en el ejército se preocupen por el bienestar de los soldados» ya que lo que realmente hacen es «obstaculizar el conflicto de intereses entre éstos y el ejército». Un comentario como éste, conduce a recordar que teorizar sobre la realidad de un contexto organizacional requiere basarse en la evidencia. La argumentación del autor de que la psicología de la salud en el ejército (o el CSF) puede interferir en los intereses de los soldados no es clara y tampoco aporta ninguna razón de cómo puede suceder lo que plantea. Por otra parte, hay que recordar que el debate se ha planteado en un país en el que la milicia no es obligatoria sino una profesión voluntaria, amparada por un sistema de bienestar sujeto a medidas de tipo administrativo, médico o forense (ver citas ${ }^{69-71}$ ). Por lo tanto, apelar a teorías de la psicología social (dilema del prisionero, dilemas sociales) para afirmar que la psicología de la salud en el ejército impide los intereses de los soldados, es discutible y no está demostrado. En todo caso, el argumento de Krueger se podría entender como un estresor laboral del psicólogo militar y asociado al conflicto de rol al que este profesional se puede llegar a enfrentar en casos puntuales cuando le hacen demandas incompatibles entre sí, como por ejemplo: Aplicar tratamientos psicoterapéuticos a un soldado que, en realidad, quiere dejar de serlo; informar que un soldado presenta psicopatología real mientras la cadena de mando opina que dicho soldado es un simulador, o que un soldado disimula psicopatología cuando los jefes creen que es apto; demandarle que haga programas de prevención de salud mental pero no proporcionarle los medios, el tiempo o el espacio necesarios para hacerlo; solicitarle tareas que, desde su rol de psicólogo, considera irrelevantes y que, además, le impiden realizar otras fundamentales de psicología; o -aunque esto ya no sucede- exigirles que asuman el rol de mando a la vez que asumen el rol de psicó- logo con los subordinados. En los casos en los que el soldado se plantee dejar el ejército, el psicólogo podrá ayudarle a canalizar sus intereses, bien adaptándose, bien tomando la decisión de solicitar la baja, en lugar de obstaculizarlos como apunta Krueger. En definitiva, por un lado, los psicólogos militares pueden fomentar la resolución de los dilemas sociales de los soldados así como reducir las consecuencias negativas de los conflictos de roles de éstos y, por otro, el conflicto de rol del psicólogo militar podrá afectar a su salud, bienestar y satisfacción, lo cual no tiene porqué dañar su ética profesional.

El mismo autor, Krueger ${ }^{10}$, plantea que en el monográfico «se justificaba el compromiso con el ejército argumentando que la psicología científica ha sido relevante sobre todo en las guerras mundiales $\left.{ }^{26}\right\rangle$. Sin embargo, deducir que lo que pretendían los autores cuando relataban el pasado de la psicología en las grandes guerras era justificarse, es una interpretación que va más allá del propio texto. Describir el pasado histórico del tema que se trataba en el monográfico, no sólo parece ilustrativo sino que puede ser habitual y apropiado para un artículo de un especial dedicado a un único asunto.

Krueger ${ }^{10}$ también afirma que «traer la psicología al ejército es usar las herramientas de la psicología para una industria». Sin embargo, no presenta ninguna razón objetiva por la que a los militares se les tenga que negar lo que la psicología puede ofrecer. No explica, desde la ética profesional, por qué la psicología se puede practicar en otras industrias (como la salud, la educación, el deporte, el trabajo y las organizaciones, etc.) pero no en la militar. Surge la duda de si este mismo argumento lo extrapolaría a otras profesiones -como la medicina, enfermería, veterinaria, farmacia, odontología, etc.- que también se ejercen en los ejércitos, o si, para Krueger, sólo es la psicología la que no debe asociarse con lo castrense.

Phipps ${ }^{9}$ emplea un lenguaje muy sutil cuando se pregunta «si la psicología puede hacer mejores soldados», «si se puede usar la resiliencia y la inoculación al estrés para inmunizar a los soldados contra el estrés de la guerra» y cuando afirma que «la pretensión del CSF es crear soldados psicológicamente invulnerables». Los programas de promoción de la salud mental, cabe decir, pretenden fomentar los factores de protección y enseñar estrategias de afrontamiento al estrés, no hacer mejores personas. Lo que es de aplicación a contextos militares. Se ha demostrado que la capacidad de hacer frente al estrés -o la resiliencia- mejora con programas psicológicos ${ }^{72}$, así como que los programas específicos de resiliencia muestran efectos positivos a largo $\mathrm{plazo}^{73}$. Lo que no significa que la psicología haga personas invulnerables e inmunes a la guerra. De hecho, estos programas, desarrollados en los ejércitos, brindan la ocasión a los militares de hablar de experiencias dolorosas en un contexto humanizado, en donde también se explica la importancia, para la salud mental, de reconocer los problemas psicosociales cuando aparecen, de compartirlos y de buscar ayuda ${ }^{42}$. Esta metodología empleada, en parte, para hacer frente al estigma, se aleja de presentar a los militares como guerreros invulnerables.

Dyckman ${ }^{11}$ interpreta con catastrofismo el CSF usando argumentos de política exterior y luego se pregunta «jen qué casos está justificado que se pueda ayudar a alguien a sentirse mejor por matar?» mientras omite que los soldados americanos desplegados (y sus familias) se exponen con frecuencia a la muerte 
en los despliegues y a otros importantes estresores psicosociales (ver informes de los MHAT, 2003- $2011^{74} \mathrm{y} \mathrm{cita}^{75}$ ) y que todo ello son riesgos laborales asociados a un trabajo desarrollado para el Gobierno y la Nación.

Además, los interrogatorios y la alusión a las torturas que este mismo autor incluyó en las críticas hacia la unión entre la psicología y lo militar merecen una réplica exclusiva. Primero, la decisión de torturar o no en un interrogatorio está muy lejos de depender de los psicólogos en general y los psicólogos militares en particular. Segundo, las torturas en los interrogatorios son mucho más antiguas que la psicología. Tercero, los interrogatorios no representan, en absoluto, la agenda de la psicología militar $\mathrm{y}$, por último, ni las torturas ni los interrogatorios tienen nada que ver con los programas de entrenamiento de la salud mental en los ejércitos. Cabe añadir que Dyckman ${ }^{11}$ no cita de qué fuente extrae esta información, cuestión relevante teniendo en cuenta la trascendencia del comentario y que el papel de la psicología o los psicólogos en los interrogatorios ha sido analizado por la APA concluyendo que, en ningún caso, este organismo de representación de la psicología haya apoyado o apoye las torturas ${ }^{76}$.

Ante la comparación del programa con investigaciones nazis, la acusación de que el CSF carece de ética y el comentario de que «la única preocupación, en el especial de la APA, fue que no era ético negar el CSF a los soldados $»^{8}$, habría que destacar (además de la contrarréplica de Seligman ${ }^{30}$ ) que, cuando se conocen programas de entrenamiento psicosocial y promoción de la salud mental eficaces y no se aplican a una población de riesgo, podrían surgir acusaciones deontológicas a la profesión y judiciales a la organización y a la cadena de mando (p. ej. ver caso de demanda colectiva interpuesta al Ministerio de Defensa del Reino Unido descrito por Greenberg y Jones ${ }^{42}$ ).

Por otra parte, evitar que se produzca el trauma para impedir que aparezcan casos de TEPT, como plantea Dickman ${ }^{11}$, es una estrategia preventiva necesaria y, seguramente, tenida en cuenta en las decisiones del Mando. Sin embargo, no se pueden evitar todos los riesgos en las profesiones que, por definición, están asociadas con altos niveles de riesgo físico y psicológico porque, entonces, perderían su razón de ser. Dado que la eliminación o la reducción de algunos estresores asociados a la profesión militar es imposible, la única alternativa de cara a fomentar la salud y el bienestar de este personal, consiste en aumentar la capacidad de afrontamiento a dichos estresores. Esto es, en definitiva, el objetivo del CSF.

\section{CONCLUSIONES}

Tal y como plantean las réplicas al monográfico de enero de 2011 de la revista de la APA, la confianza depositada por líderes políticos y militares de los Estados Unidos en la psicología, lejos de agradar a todos, han provocado un debate entre profesionales. La polémica suscitada es interesante y necesaria, llevando a la reflexión y el diálogo. Con ella se demuestra que la psicología es una ciencia dinámica y está abierta a discusiones éticas sobre decisiones importantes que afectan a la salud y bienestar de las personas.
Las réplicas a Seligman y sus colaboradores están relacionadas, principalmente, con tres asuntos. El primero guarda relación con ideologías políticas, el segundo con la unión entre psicología y ejército y, el tercero, con la psicología positiva aplicada al personal militar. Pero estos tres asuntos se mezclan, desviándose del objetivo que persiguen las réplicas y creando, por tanto, cierta confusión. En ocasiones, parece que el fondo del asunto no es tanto la psicología de Seligman y sus colaboradores, como la defensa de una determinada posición política.

Al margen de las ideologías personales, los críticos al CSF plantean un debate moral sobre si la psicología debe aplicarse al personal militar, siendo el cliente una «industria» bélica y no los soldados individualmente. Sin embargo, ante el rechazo a la unión entre psicología y ejército, no aportan ninguna solución. Ante esto, surge la duda de si su propuesta es que los psicólogos no deberían trabajar en contextos militares, creando así una paradoja. Si se eliminara la psicología de la organización militar, supondría una dejación de las funciones de ésta y, por tanto, una falta de ética institucional, pudiendo surgir acusaciones deontológicas a la profesión y judiciales a la organización y a la cadena de mando (como ya ha ocurrido en algunos países). Por otra parte, la psicología militar tiene sus funciones y principios bien establecidos (ver citas ${ }^{77-79}$ ), tanto como la psicología en otras organizaciones o tanto como otras profesiones en los ejércitos. Además, hay miles de psicólogos militares en el mundo comprometidos con la ética de su profesión. En todo caso, más que un problema de ética profesional se puede entender como un riesgo laboral de origen psicosocial, originado por exponer a psicólogos militares ante demandas incompatibles entre sí o, lo que es lo mismo, posicionarlos en un conflicto de rol.

La psicología positiva es, todavía, una rama joven de la psicología y puede que, en ocasiones, no comprendida o mal empleada. No obstante, algunas cuestiones del módulo psicológico del CSF se construyeron basándose en la evidencia de aspectos positivos, la mayoría ya planteados por la psicología, antes de que éstos se incluyeran en el área de la psicología positiva (para una recopilación de la literatura sobre psicología positiva ver $\left.\operatorname{citas}^{64,80-85}\right)$.

Independientemente de los conflictos bélicos a los que se enfrente un país y al margen de las ideologías políticas de los psicólogos militares -que pueden ser variadas- el trabajo de éstos debe basarse, siempre, en el código deontológico. Tanto si las guerras son entendidas y apoyadas por la sociedad, los medios de comunicación y el propio psicólogo, como si no lo son, los soldados y sus familias merecen lo mejor de la psicología. Negar que la psicología pueda tener las mismas funciones, relacionadas con la salud y el bienestar, en la organización militar que en otras organizaciones, es negar la realidad. Otra cuestión es la de emplear la psicología con fines manipulativos o a favor, sólo, de los gobiernos y no de los usuarios. Cuestiones, estas últimas, que deben perseguirse y denunciarse cuando haya pruebas objetivas que lo demuestren.

\section{BIBLIOGRAFIA}

1. SCImago (2007). SJR — SCImago Journal \& Country Rank. Retrieved December 18, 2012, from http://www.scimagojr.com 


\section{La polémica sobre el entrenamiento psicológico del Comprehensive soldier fitness}

2. Harms PD, Herian MN, Krasikova DV, Vanhove A y Lester PB. The comprehensive soldier and family fitness program evaluation report \# 4: Evaluation of resilience training and mental and behavioral health outcomes. Monterrey, CA: Office of the Deputy Under Secretary of the Army, 2013.

3. Silgo MG. Revisión de programas de resiliencia basados en la evidencia en los ejércitos. Sanidad Militar 2013;69(3):182-194

4. Silgo, M.G. El papel de la resiliencia en las Fuerzas Armadas. En D. Donoso Rodriguez (Coord.): Psicología en las Fuerzas Armadas. Ministerio de Defensa, Secretaria General Técnica, 2012a. http://publicacionesoficiales.boe.es/

5. Silgo MG. Intervenciones de resiliencia en contextos militares. En MP Bardera (Coord.): Riesgos Psicosociales y Resiliencia en Cuerpos y Fuerzas de Seguridad. Jornadas celebradas el 12/12/12. Madrid: UNED, 2012b.

6. Silgo MG y Castellanos Díaz JL. Preparación psicológica a través de programas ad oc. Revista Ejército 2013;863:63-68.

7. Silgo MG y Bardera P. Resiliencia: La defensa psicológica. Revista Española de Defensa, 2010;266:55-56.

8. Eidelson R, Pilisuk M y Soldz S. The dark side of Comprehensive Soldier Fitness. American Psychologist 2011;66(7):643-644.

9. Phipps S. Positive psychology and war: An oxymoron. American Psychologist 2011;66(7):641-642

10. Krueger JI. Shock without awe. American Psychologist 2011;66(7):642-643.

11. Dyckman J. Exposing the glosses in Seligman and Fowler's (2011) straw-man arguments. American Psychologist 2011;66(7):644-645.

12. Campbell Quick J. Missing: critical and skeptical perspectives on Comprehensive Soldier Fitness. American Psychologist 2011;66(7):645.

13. Casey GW. Comprehensive Soldier Fitness: A vision for psychological resilience in the U.S. Army. American Psychologist 2011;66(1):1-3

14. Seligman MEP. The President's address. APA 1998 Annual Report. American Psychologist 1999;54:559-562.

15. Organización Mundial de la Salud (1948). Preámbulo de la Constitución de la Organización Mundial de la Salud, adoptada por la Conferencia Sanitaria Internacional, Nueva York, 19 de junio- 22 de julio de 1946, firmada el 22 de julio de 1946. Entró en vigor el 7 de abril de 1948

16. Organización Mundial de la Salud (1986). International Conference on Health Promotion. Ottawa Charter for Health Promotion, 17-21, nov-1986. Ottawa, Ontario, Canada

17. Organización Mundial de la Salud (1997). IV International Conference on Health Promotion. New Players for a New Era: Leading health promotion into the 21st century. The Jakarta Declaration on Health Promotion in the 21st. Century, July 1997. Jakarta, Indonesia.

18. Declaración Europea de Salud Mental. (2005). Declaración de los Ministros de Sanidad de los Estados Miembros en la Región Europea de la OMS, reunidos en Conferencia Ministerial celebrada en Helsinki del 12 al 15 de enero de 2005. http://www.msps.es/organizacion/sns/planCalidadSNS/saludMental.htm

19. Libro Verde (2005). Mejorar la salud mental de la población: Hacia una estrategia de la Unión Europea en materia de salud mental. Comisión de las Comunidades Europeas, Bruselas. http://europa.eu/documentation/officialdocs/green-papers/index_es.htm

20. Organización Internacional del Trabajo. (2010). Riesgos emergentes y nuevos modelos de prevención en un mundo de trabajo en transformación. Ginebra.

21. Ministerio de Sanidad y Consumo (2007). Estrategia en Salud Mental del Sistema Nacional de Salud (2006)

22. Ministerio de Sanidad, Política Social e Igualdad (2011). Estrategia en Salud Mental del Sistema Nacional de Salud (2009-2013).

23. Barnett MD. Congress Must Recognize the Need for Psychological Resilience in an Age of Terrorism. Families, Systems, \& Health 2004;22(1):64-66.

24. National Security Research Division. (2012). Annual Report 2011-2012. Santa Monica, CA: RAND Corporation, National Defense Research Institution

25. Hoge CW, Castro CA, Messer SC, McGurk D, Cotting DI y Koffman RL. Combat duty in Iraq and Afghanistan, mental health problems, and barriers to care. New England Journal of Medicine 2004;351:13-22.

26. Seligman MEP y Fowler RD. Comprehensive Soldier Fitness and the future of psychology. American Psychologist 2011;66(1):82-86.

27. Cornum RL, Matthews MD y Seligman MEP. Comprehensive Soldier Fitness: Building resilience in a challenging institutional context. American Psychologist 2011;66(1): 4-9.

28. Ritter LA y Lampkin SM. Prevention and mental health promotion in community mental health. En L.A. Ritter y S.M. Lampkin (Eds.), Community Mental Health. Sudbury, MA: Jones \& Bartlett Learning. 2012.
29. González-Camino G y Osca Segovia A. Estrés y salud laboral. En A. Osca Segovia (Ed.), Psicología de las Organizaciones. Madrid: Sanz y Torres. 2004:301-344

30. Seligman MEP. Helping American soldiers in time of war: Reply to comments on the Comprehensive Soldier Fitness special issue. American Psychologist 2011;66(7):646-647

31. Coyne JC y Tennen H. Positive psychology in cancer care: Bad science, exaggerated claims, and unproven medicine. Annals of Behavioral Medicine 2010;39:16-26.

32. Brunwasser SM, Gillham JE y Kim ES. A meta-analytic review of the Penn Resiliency Program's effect on Depressive Symptoms. Journal of Consulting and Clinical Psychology, 2009;77(6);1042-1054

33. Williams A, Hagerty BM, Yousha SM, Horrocks J, Hoyle KS y Liu D. Psychosocial effects of the boot strap intervention in Navy recruits. Military Medicine 2004; 169(10):814-822

34. Gottman JM, Gottman JS y Atkins C. The Comprehensive Soldier Fitness program: Family skills component. American Psychologist 2011;66(7);52-57.

35. Aspinwall LG y Tedeschi RG. The Value of Positive Psychology for Health Psychology: Progress and Pitfalls in Examining the Relation of Positive Phenomena to Health. Annals of Behavioral Medicine 2010;39:4-15.

36. Smith SL. Could Comprehensive Soldier Fitness have iatrogenic consequences? A commentary. The Journal of Behavioral Health Services \& Research 2013;40(2):242-246.

37. Lester PB, Harms PD, Bulling DJ, Herian MN y Spain SM. Evaluation of relationships between reported resilience and soldier outcomes: Report \#1: Negative outcomes (suicide, drug use, and violent crime) (Tech. Rep. 1). (2011a, February).

38. Lester PB, Harms PD, Bulling DJ, Herian MN y Spain SM. Evaluation of relationships between reported resilience and soldier outcomes: Report \#2: Positive performance outcomes in officers (promotions, selections, and professions). (Tech. Rep. 2). (2011b, April).

39. Lester PB, Harms PD, Herian MN, Krasikova DV y Beal SJ. The Comprehensive Soldier Fitness program evaluation: Report \#3: Longitudinal Analysis of the impact of Master Resilience Training on self-reported resilience and psychological health data. (Tech. Rep. 3). (2011, Diciembre).

40. Remor E. Contribuciones de la psicología positiva al ámbito de la psicología de la salud y medicina conductual. En C. Vázquez y G. Hervás (Eds.): Psicología Positiva Aplicada. Bilbao: Desclée de Brouwer. 2008.

41. McGeorge T, Hughes JH y Wessely S. The MOD PTSD decision: A psychiatric perspective. Occupational Health Review 2006;122:21-28.

42. Greenberg $\mathrm{N}$ y Jones N. Optimizing mental Health support in the military: The role of peers and leaders. En A.B. Adler, P.D. Bliese y C.A. Castro (Eds.), Deployment Psychology. Washington DC: American Psychological Association. 2011: 69-101.

43. Tedeschi RG y Calhoun LG. The Posttraumatic Growth Inventory: Measuring the positive legacy of trauma. Journal of Traumatic Stress 1996;9:455-472.

44. Tedeschi RG y McNally RJ. Can we facilitate posttraumatic growth in combat veterans? American Psychologist 2011;66(1):19-24

45. Park N. Military children and families: Strengths and challenges during peace and war. American Psychologist 2011;66(1):65-72.

46. Meredith LS, Sherbourne CD, Gaillot S, Hansell L, Ritschard HV, Parker AM y Wrenn G. Promoting psychological resilience in the U.S. military. Santa Monica, CA: RAND Corporation. 2011

47. Castro CA y Adler AB. Military mental health training: building resilience. En S.M. Southwick, B.T. Litz, D. Charney y M.J. Friedman (Eds.), Resilience and Mental Health: Challenges Across the Lifespan. Cambridge, UK: Cambridge University Press. 2011:323-339.

48. Bliese PD, Adler AB y Castro CA. Research-Based preventive mental health care strategies in the military. En A.B. Adler, P.D. Bliese y C.A. Castro (Eds.), Deployment Psychology. American Psychological Association: Washington DC. 2011:103-124.

49. Warner CH, Appenzeller GN, Breitbach JE, Mobbs A y Lange JT. The care framework: the broadening of mental health services in a deployed environment. En A.B. Adler, P.D. Bliese y C.A. Castro (Eds.), Deployment Psychology. Washington DC: American Psychological Association. 2011:35-68.

50. Nash WP, Krantz L, Stein N, Westphal RJ y Litz B. Comprehensive soldier fitness, battlemind, and the stress continuum model: Military organizational approaches to prevention. En J.I. Ruzek, P.P. Schnurr, J.J. Vasterling y M.J. 
Friedman (Eds.), Caring for veterans with deployment-related stress disorders: Irak, Afganistan, and beyond. Washington DC: American Psychological Association. 2011:193-214

51. Victoria DA. Zen at war. New York: Weatherhill. 1997.

52. Victoria DA. Zen war stories. London: Routledge. 2003.

53. Donahue MJ. Intrinsic and extrinsic religiousness: Review and meta-analysis. Journal of Personality and Social Psychology 1985;48:400-419.

54. Emmons RA, Cheung $\mathrm{C}$ y Tehrani K. Assessing spirituality through personal goals: Implications for research on religion and subjective well-being. Social Indicators Research 1998;45:391-422.

55. Ano GG y Vasconcelles EB. Religious coping and psychological adjustment to stress: A meta-analysis. Journal of Clinical Psychology 2005;61:461-480.

56. Mahoney A, Pargament KI, Cole B, Jewell T, Magyar GM, Tarakeshwar N, Phillips R. A higher purpose: The sanctification of strivings in a community sample. International Journal for the Psychology of Religion 2005;15:239-262.

57. Harris JI, Erbes CR, Engdahl BE, et al. Christian religious functioning and trauma outcomes. Journal of Clinical Psychology 2008;64:17-29.

58. Stouffer SA, Lumsdaine AA, Lumsdaine MH, Williams RW Jr., Smith MB Janis IL, ... Cotrell LS Jr. The American soldier: Combat and its aftermath (Vol. 2). New York: Wiley. 1949.

59. Witvliet CVO, Phillips KA, Feldman ME y Beckham JC. Posttraumatic mental and physical health correlates of forgiveness and religious coping in military veterans. Journal of Traumatic Stress 2004;17:269-273.

60. Fontana A y Rosenheck R. Trauma, change in strength of religious faith, and mental health service use among veterans treated for PTSD. Journal of Nervous and Mental Disease 2004;192:579-584.

61. Benda BB, DiBlasio FA y Pope SK. Spiritual well-being, relationships, and work satisfaction in the treatment of homeless veterans with alcohol and other drug problems. Alcoholism Treatment Quarterly 2006;24:109-124.

62. Pargament KI y Mahoney A. Spirituality: The search for the sacred. En S.J. Lopez y C.R. Snyder (Eds.), Oxford Handbook of Positive Psychology. New York: Oxford University Press. 2009:611-619.

63. Pargament KI y Sweeney PJ. Buiding spiritual fitness in the Army. American Psychologist 2011;66(1):58-64

64. Adler AB, Bliese PD y Castro CA. An introduction to deployment psychology. En A.B. Adler, P.D. Bliese y C.A. Castro (Eds.), Deployment Psychology. American Psychological Association: Washington DC. 2011:3-13.

65. Sharpley JG, Fear NT, Greenberg N, Jones M y Wessely S. Predeployment stress briefing: does it have an effect? Occupational Medicine 2007;58:30-34.

66. Poundstone W. El dilema del prisionero. Madrid: Alianza. 2009.

67. Pruitt DG y Kimmel MJ. Twenty years of experimental gaming: critique, thesis, and suggestions for the future. Annual Review of Psychology 1977;28:641-651.

68. Dawes RM. Social dilemas. Annual Review of Psychology 1980;31:169-193.

69. Silgo MG. La simulación de psicopatología en los ejércitos desde la revisión de investigaciones empíricamente validadas. En D. Donoso Rodriguez (Coord.):
Psicología en las Fuerzas Armadas. Ministerio de Defensa, Secretaria General Técnica. (2012c). Retrieved from http://publicacionesoficiales.boe.es/

70. Silgo MG y Robles Sánchez JI. Estudio de las bajas temporales para el servicio por motivos psicológicos: mejoras en la detección de psicopatología y simulación de trastorno mental en las Fuerzas Armadas. Sanidad Militar 2010;66(3):154-162.

71. Silgo MG, Bardera Mora MP, Sánchez Sánchez JM y Robles Sánchez JI. Simulación de psicopatología en contextos militares. En H. González Ordi, P. Santamaría Fernández y P. Capilla Ramírez (Coord.), Estrategias de Detección de la Simulación: Un Manual Clínico Multidisciplinar. Madrid: TEA Ediciones. 2012:703-728.

72. Seligman MEP, Steen TA, Park N y Peterson Ch. Positive psychology progress: Empirical validation of interventions. American Psychologist 2005;60(5):410- 421.

73. Duckworth AL, Steen TA y Seligman MEP. Positive Psychology in Clinical Practice. Annual Review of Clinical Psychology 2005;1:629-651.

74. Retrieved from http://www.behavioralhealth.army.mil/research/index.html

75. Castro CA, Hoge CW y Cox AL. Battlemind training: Building Soldier resiliency. En Human Dimensions in Military Operations- Military Leaders'Strategies for Addressing Stress and Psychological Support. Meeting Proceedings RTO-MP-HFM-134, Parer 42. Neuilly-sur-seine, France: RTO. 2006:42-1 - 42-6.

76. Behnke SH y Moorehead-Slaughter O. Ethics, human rights and interrogations. En J.H. Laurence y M.D. Matthews (Eds.), The Oxford Handbook of Military Psychology. New York: Oxford University Press. 2012:50-62.

77. Laurence JH y Matthews MD. The handbook of military psychology: An introduction. En J.H. Laurence y M.D. Matthews (Eds.), The Oxford Handbook of Military Psychology. New York: Oxford University Press. 2012:1-3.

78. Matthews MD y Laurence JH. Military psychology: closing observations and a look forward. En J.H. Laurence y M.D. Matthews (Eds.), The Oxford Handbook of Military Psychology. New York: Oxford University Press. 2012:400-404

79. Orden Ministerial 141/2001, de 21 de junio, por la que se establecen las Funciones y Estructura de la Psicología Militar.

80. Fernández-Abascal EG (Coor.). Emociones positivas. Madrid: Pirámide. 2009.

81. Aspinwall LG y Staudinger UM (Eds.) Psicología del potencial humano. Barcelona: Gedisa. 2007.

82. Vázquez C y Hervás G. Psicología Positiva Aplicada. Bilbao: Desclée de Brouwer. 2008

83. Vázquez C y Hervás G. La ciencia del bienestar: Fundamentos de una psicología positiva. Madrid: Alianza. 2009.

84. Reich JW, Zautra AJ y Hall JS (Eds.). Handbook of adult Resilience. New York: Guilford Press. 2010.

85. Southwick SM, Litz BT, Charney D y Friedman MJ (Eds.). Resilience and mental health: Challenges across the lifespan. Cambridge: Cambridge University Press. 2011. 